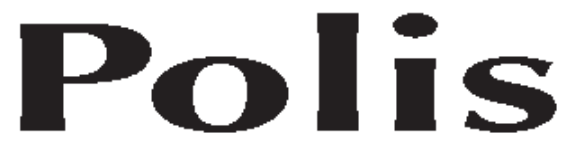

\title{
Los agrocombustibles y el mito de las tierras marginales
}

\author{
Teresa Anderson** \\ Helena Paul \\ Guadalupe Rodríguez
}

\begin{abstract}
Resumen: Las autoras debaten que las políticas de producción de agrocombustibles estén destinadas a implementarse efectivamente sobre tierras "marginales", pues que en realidad son tierras vitales para campesinos o bien comunitarias. Recuerda que la tierra marginal para algunos es vital para otros, que como siempre verán restringidas tierras y fuentes de vida, que pasarán a manos de los grandes empresarios de agrocombustibles. Advierte que esto traerá también consecuencias medioambientales negativas. Concluye cuestionando algunos mitos sobre sus efectos sobre la producción y el empleo, y anexa cuatro estudios de caso.
\end{abstract}

Palabras clave: agrocombustibles, tierras marginales, medioambiente, campesinos

\section{The agrocombustibles and the myth of marginal lands*}

Abstract: The authors debate that agrocombustibles production policies are actually destined to «marginal», lands, since in reality they constitute vital lands for peasants or belong to local natives. He points out that marginal land for some is vital for others, the ones that, as usual, will see restricted their lands and sources of life, that will pass to agrocombustibles entrepreneurs. He then notifies that this will also bring negative environment consequences. Finally he questions some myths on its effects on production and employment. Four case studies are also enclosed.

Key words: agrocombustibles, marginal lands, environment, rural

Recibido 20.11 .08

Aceptado 09.12.08

$* * *$

Marginales, sin uso, degradados, poco utilizados, dormidos, páramos y terrenos de cultivo abandonados, son algunos de los términos usados para referirse a lo que está siendo

\footnotetext{
* Documento elaborado por The Gaia Foundation, Biofuelwatch, African Biodiversity Network, Salva La Selva, Watch Indonesia y EcoNexus, Septiembre 2008. Traducción: Traductores sin Fronteras (TSF), alugris@uvigo.es

*** The Gaia Foundation, Email: teresa@ gaianet.org

**** EcoNexus, Oxford. Reino Unido. Email: h.paul@gn.apc.org

***** Salva la Selva, Berlín, Alemania. Emial: guadalupe@regenwald.org
} 
promocionado como la "solución" al impacto que tienen los cultivos destinados a agrocombustibles.

En parte para responder a las acusaciones de que los agrocombustibles (también conocidos como biocombustibles) compiten con la producción de alimentos, algunos políticos propusieron que los agrocombustibles deberían ser plantados en terrenos considerados como marginales o sin uso. Se dice que hay millones de hectáreas de este tipo de tierras alrededor del mundo, especialmente en África, que no tendrían importancia para la biodiversidad o para la eliminación de carbono, y que tampoco tendrían relevancia para la producción de alimentos ni para garantizar el sustento de la población. Algunos proponen que plantar agrocombustibles en las "tierras marginales" puede ser extremadamente positivo, aportando ingresos a las comunidades locales y suministrando una alternativa en el mercado a los combustibles fósiles. Se ha sugerido incluso que debería haber incentivos para usar las llamadas tierras marginales, como por ejemplo licencias para emitir más CO2.

Existe la idea muy extendida de que los países en desarrollo tienen bastantes extensiones de páramos, a la espera de que alguien les dé un uso productivo. Pero si miramos más detenidamente estas tierras "marginales" la realidad es muy diferente, ya que en muchos casos, las tierras definidas como "marginales", "páramos" o "sin uso" son vitales para el sustento de pequeños campesinos, pastores, mujeres y pueblos indígenas. Eso a lo que el gobierno o las transnacionales llaman tierras "marginales" son de hecho tierras comunales o tradicionales que han estado en uso durante generaciones, y que no son propiedades privadas, o no están en producción agrícola intensiva. Las vidas de las personas que habitan en estas tierras son ignoradas a menudo.

Las comunidades que usan estas tierras ricas en biodiversidad para producir sus alimentos, sus ingresos, o para el pastoreo o la medicina no aprecian el hecho de que su existencia sea negada. Tampoco están siempre de acuerdo en que la conversión de sus tierras para la producción de agrocombustibles aportará beneficios para su "desarrollo". Ante la evasiva promesa de una escasa compensación o unos trabajos mal pagados y discontinuos en las plantaciones de agrocombustibles, muchas de estas comunidades preferirían continuar viviendo como campesinos, pastores o cazadores-recolectores, antes que ser desalojados y desterrados para siempre de sus tierras.

La evidencia indica que realmente existen muy pocas tierras auténticamente "marginales", o por lo menos ninguna que se corresponda con las tierras abandonadas, vacías y sin uso que nos hacen imaginar. Las políticas de agrocombustibles basadas en tierras "marginales" deben ser por ello seriamente replanteadas. 


\section{"Las tierras marginales" en las políticas de agrocombustibles}

El informe británico Gallagher sostiene que los impactos negativos indirectos de los agrocombustibles se pueden evitar si se cultivan en tierras marginales. "La producción de biocombustibles debe apuntarse a tierras marginales y sin uso y al aprovechamiento de desperdicios y residuos". En esto sigue las recomendaciones dadas por la Royal Society, cuyo informe "Biocombustibles sostenibles: perspectivas y retos" también solicita decisiones políticas... para cambiar paulatinamente esos cultivos a tierras de baja biodiversidad, tierras marginales o tierras abandonadas.

Incluso el ampliamente difundido estudio hecho por Fargione et al. que informa de que los actuales agrocombustibles acumulan una "deuda de carbono" de décadas o siglos", concluye sin embargo que: "Las tierras agrícolas degradadas y abandonadas se podrían usar para el cultivo de plantas nativas perennes para la producción de agrocombustibles, lo que podría ahorrar la destrucción de ecosistemas nativos y reducir las emisiones de carbono".

Términos como "tierras marginales" han alcanzado no solamente el debate científico sino también más ampliamente el discurso político sobre los agrocombustibles. El autor de la Ley de Biocombustibles de Filipinas, senador Zuburi, por ejemplo, promovió "los cultivos de biocombustibles en tierras marginales" en la Conferencia Mundial sobre Biocombustibles $^{1}$ en el 2008. El gobierno de la India apoya la conversión de hasta 14 millones de hectáreas de las llamadas "tierras de deshecho" para cultivar jatrofa para biodiesel. El presidente de Mozambique, Armando Guebuza, hizo un llamamiento para que los biocombustibles creciesen en tierras "marginales" para que no compitiesen con la producción de alimentos. $^{2}$

En la Unión Europea, el informe del Borrador de la Directiva de Energías Renovables hecho por el Relator del Comité de Industria, Investigación y Energía, Claude Turmes, propone una definición de "tierras sin uso, degradadas y marginales", que es considerada como adecuada para la producción de bioenergía, incluyendo los cultivos para agrocombustibles. Y tanto los principios y criterios de la Mesa Redonda de Aceite de Palma Sostenible RSPO, como el borrador de la Mesa Redonda sobre Biocombustibles Sostenibles, apoyan el uso de tierras "marginales, degradadas o previamente desmontadas".

\section{¿Dónde están las tierras marginales?}

¿Dónde se encuentran las tierras marginales y cuál es su extensión? Un estudio hecho

\footnotetext{
${ }^{1}$ Ver: http://www.gmanews.tv/story/95762/Plant-biofuel-crops-in-marginal-land-Zubiri

${ }^{2}$ Ver: http://allafrica.com/stories/200802191141.html
} 
en 2006 por David Tilman et al. estima un alto potencial de bioenergía a partir del cultivo de bajo coste de pastos nativos perennes biodiversos. El autor sugiere que esto podría ser una fuente de agrocombustibles de segunda generación con saldo negativo de carbono. No investigaron sin embargo la cantidad de "tierras marginales" existentes. En lugar de ello, utilizaron datos anteriores de otros autores acerca de cuántas "tierras de cultivo abandonadas" había disponibles - por lo menos 500 millones de hectáreas, dicen.

El término "tierra marginal" parece haber sido combinado con el concepto de "terrenos de cultivo abandonados", que se encuentra en el centro de muchos de los "estudios de viabilidad de la bioenergía" que proporcionan las "bases científicas" para las políticas de biocombustibles de los gobiernos. Muchas de estas, a su vez, toman datos de estimaciones generales sobre la cantidad de tierra que podría haber disponible no para agrocombustibles sino como sumideros de carbono, lo que incluye a las plantaciones de árboles — que es donde se origina la cifra de 500 millones de hectáreas. Contemplan las "tierras de cultivo abandonadas", que incluyen grandes áreas de tierras donde se han destruido las selvas tropicales para crear plantaciones y establecer ganadería y tierras donde la degradación del suelo y el agotamiento de los recursos hídricos hacen difícil la agricultura. Como se afirma en el artículo de Goeren Berndes, que ha revisado 17 estudios sobre la viabilidad de la bioenergía: "la tierra calificada como degradada suele ser la base del sustento de las poblaciones rurales".

Un ejemplo de cómo se hacen las estimaciones sobre "tierras de cultivo abandonadas" disponibles para agrocombustibles es un estudio de 2008 de Christopher Field et al. que sugiere que existen 386 millones de hectáreas de este tipo de superficies. Se considera "tierra abandonada" a cualquier terreno que haya sido cultivado con posterioridad a 1700 y que las imágenes de satélite no identifiquen actualmente como "tierra de cultivo", a no ser que se haya reforestado o que forme parte de asentamientos urbanos. No existen estudios críticos que indiquen si estas imágenes de satélite están ignorando a las pequeñas propiedades de las comunidades nativas, pero es evidente que cuando se define el concepto de "tierra de cultivo abandonada" se ignoran usos de la tierra, como el destinado a pastos.

Esta omisión se encuentra en el centro de todos los estudios que defienden que existen grandes áreas de terreno disponibles para la producción de agrocombustibles, o que pueden ponerse a disposición, incluyendo estudios que se basan en datos no sólo actuales, sino también en la previsión de las tierras que se abandonarán en el futuro, tierras que pueden ser "liberadas" para las plantaciones de biomasa. Con muchos de estos estudios, enormes áreas de tierra de África, América Latina y Asia, especialmente pastos, se definen como tierras "infrautilizadas". 


\section{Personas olvidadas - pastores, pequeños campesinos, indígenas y mujeres}

Antes de considerar lo que se debería cultivar en las tierras marginales, debemos definir qué es "marginal". La tierra que a una persona le puede parecer "marginal" puede ser un recurso vital imprescindible para otra. Aunque a un extranjero le puede parecer desocupada o infrautilizada, esta tierra a menudo proporciona alimentos, combustible, medicinas y materiales de construcción a las comunidades locales. Puede tratarse de tierras comunales utilizadas por tales comunidades durante generaciones, aunque no tengan un título de propiedad formal. La fragilidad de estas tierras puede significar que son cultivadas rotativamente en períodos largos, volviendo a cultivar ciertas áreas tras dejarlas en barbecho durante varios años. Estas tierras pueden ser sagradas para las comunidades locales e imprescindibles para proteger los recursos hídricos.

El Informe Gallagher parece defender que "tierra infrautilizada y marginal" es sinónimo de pastizales. Sin embargo, pocos pastizales están realmente infrautilizados. Existen grandes extensiones de terreno, especialmente en África, utilizadas por ganaderos y pastores, que necesitan desplazarse a través de amplias áreas siguiendo las lluvias y pastos frescos. Los observadores externos tienden a pensar que la vida de estos pueblos podría mejorar si se estableciesen en un lugar y se les proporcionasen medios para implementar cultivos. Sin embargo, estos pueblos tienen derecho a continuar con la forma de vida que han venido manteniendo y perfeccionando a través de generaciones y que podría ser la única manera de utilizar las frágiles tierras de pastos. Las llamadas tierras marginales son también importantes para la biodiversidad y constituir una reserva de especies amenazadas o útiles.

Jonathan Davies, coordinador general de la Iniciativa Mundial para el Pastoreo Sostenible, con base en Nairobi (Kenia), comenta que "estas tierras marginales no son tantas como se supone. En África, la mayor parte de las tierras en cuestión están siendo explotadas activamente por pastores, cazadores-recolectores y algunas veces por agricultores de secano. Esto no es para decir que la bioenergía no va a tener cabida en el continente africano. Pero dada la actual permisividad en las expropiaciones de tierras o la falta de respeto por los derechos a las tierras de los habitantes de las zonas rurales de muchos países, es inevitable que van a ser los grandes inversores los que se ocupen de la producción de agrocombustibles y será a expensas de las comunidades locales”.

Si se expanden los cultivos para agrocombustibles sobre las tierras marginales, como está planificado, es más que probable que las comunidades afectadas perderán sus tierras y no recibirán ningún beneficio a cambio. Muy al contrario, se verán confinadas a áreas más pequeñas u obligadas a trasladarse a regiones aún más frágiles, una experiencia que ya han sufrido en el pasado. Al mismo tiempo, la adquisición de tierras minará las instituciones consuetudinarias y la sabiduría ecológica tradicional que asegura un uso sostenible de la 
tierra. Esto conducirá a la pérdida de biodiversidad, no sólo en las áreas en las que se realicen siembras, sino también en todo el país en general. Esto ya ha pasado en África con anterioridad, especialmente cuando la tierra se destinó a la producción de cereales, poniéndola en manos privadas o se anexionó para crear reservas.

La Organización de las Naciones Unidas para la Agricultura y la Alimentación (FAO) ha señalado también el impacto negativo que la producción de biocombustibles a gran escala tendrá sobre las mujeres de las zonas rurales. Según un informe ${ }^{3}$ de 2008 , las tierras marginales son un factor clave en la subsistencia de los habitantes empobrecidos de las zonas rurales, y frecuentemente son mujeres quienes las cultivan. A veces son las únicas tierras a las que éstas pueden acceder, ya que en muchas partes del mundo no tienen derechos de propiedad o de herencia. Para las mujeres, estas tierras pueden marcar la diferencia entre la vida y la muerte en los momentos más duros, porque saben cómo extraer de allí alimentos. Las mujeres que usan estas tierras seguramente son muy conscientes de su fragilidad, pero saben cómo utilizarlas sin degradarlas.

No es una coincidencia que en este debate se estén ignorando los medios de vida de las comunidades que no practican la agricultura intensiva y en particular de los pastores y de las mujeres. Mientras que la deforestación para la producción de agrocombustibles se ve como algo que se debería evitar, la conversión de pastos y de aprovechamientos no intensivos de tierras cultivadas en el Sur está considerada como algo esencial y deseable si la bioenergía está destinada a sustituir una cantidad significativa de los combustibles fósiles que se consumen en las sociedades industrializadas. El concepto de "tierras marginales" parece ser precisamente otro término popularizado para justificar esta estrategia generalizada de desplazamiento de los habitantes de las zonas rurales y para la industrialización de la agricultura a nivel mundial.

\section{Los cambios de uso de la tierra significan más cambio climático}

Se ha debatido mucho la cuestión del aumento de las emisiones debidas a cambios en los usos de la tierra, como por ejemplo cuando se talan bosques para establecer cultivos. Sin embargo, no se habla tanto de los cambios climáticos que se producen a nivel local y regional y que también se pueden deber a este factor. Las investigaciones del Instituto Internacional de Investigaciones Pecuarias (ILRI) y la Universidad del Estado de Michigan (MSU) muestran que en el este de África, el cambio de pastos por cultivos alteraría el clima, haciendo que algunas zonas sean más húmedas y otras más secas, con inundaciones

\footnotetext{
${ }^{3}$ Andrea Rossi y Yianna Lambrou (2008), Gender and Equity Issues in Liquid Biofuels Production-
} Minimising the Risks to Maximise the Opportunities, FAO, abril de 2008. 
y sequías más extremas. ${ }^{4}$ En muchas partes del mundo, las poblaciones ya están sufriendo cambios climáticos locales provocados por cambios en los usos del suelo, además de por el aumento generalizado de las emisiones.

\section{Los agrocombustibles en las "tierras marginales de Estados Unidos y Europa"}

En Estados Unidos y en Europa hay millones de hectáreas de terreno previamente abandonado que se están convirtiendo en monocultivos como consecuencia de la creciente presión del lobby de la agroenergía, y los precios en alza de las cosechas que los agrocombustibles han ayudado a aumentar.

En Estados Unidos, el Programa de Conservación de Reservas (CRP) se está viendo reducido y su futuro está amenazado dado que la industria del etanol y el lobby del agronegocio presionan para que se permita a los agricultores salirse de este programa sin penalizaciones. El CRP subvenciona a los agricultores que abandonan la producción y plantan pastos, arbustos y árboles para recuperar la cubierta vegetal. Está destinado especialmente a tierras "de productividad marginal" y con alto riesgo de erosión del suelo, así como "ambientalmente sensibles".

Se cree que el CRP es el programa medioambiental más exitoso de protección de la biodiversidad, reducción de la erosión del suelo, protección de las aguas dulces y control natural de las inundaciones de cuantos se desarrollan en los Estados Unidos. Según las cifras del gobierno de los Estados Unidos, evita la erosión de 408 millones de toneladas de suelo cada año; y cada año, las tierras que se acogen al CRP secuestran 21 millones de toneladas de carbono ${ }^{5}$. El $40 \%$ de las colmenas dedicadas a la producción dependen del $\mathrm{CRP}$, al igual que un gran número de especies animales y vegetales, como las aves silvestres o las de las praderas. ${ }^{6}$

En 2006 se incluyeron en el CRP 15,9 millones de hectáreas, aproximadamente un $10 \%$ de todas las tierras de cultivo de Estados Unidos. Desde entonces, se han vuelto a cultivar casi dos millones de hectáreas y se calcula que en los próximos tres años serán 4,4 millones más. Según un informe de una organización conservacionista, esta reducción prevista del CRP en sólo tres estados liberará tanto carbono como si comenzasen a circular

\footnotetext{
${ }^{4}$ CLIP (ILRI, MSU) (2008), The Effects of Climate and Land Use Changes on Climate and Agricultural Systems in Kenya, junio de 2008.

5 FAPRI (2007), "Estimating Water Quality, Air Quality and Soil Carbon Benefits of the Conservation Reserve Program", Facultad de Agricultura, Alimentación y Recursos Naturales, enero de 2007. http://www.fsa.usda.gov/Internet/FSA_File/606586_hr.pdf

${ }^{6}$ Environmental Defense Fund factsheet (2008), Circular del Fondo de Defensa Medioambiental. http://www.nativeecosystems.org/documents/CRPFactSheet 
15 millones de coches más. ${ }^{7}$ Un estudio contrastado publicado en 2008 sugiere que dedicar al cultivo de cereales para la producción de etanol unas tierras que han estado 15 años dentro del CRP supondría una "deuda de carbono" que se tardaría 48 años en pagar. ${ }^{8}$

En la Unión Europea, el 10\% de la superficie de cultivo que se dejó improductiva desde la década de 1990 se está convirtiendo con rapidez en monocultivos intensivos como consecuencia de la presión que ejerce la industria de los agrocombustibles y de los altos precios de las commodities. Desde 2007 se han vuelto a poner en producción 1,5 millones de estas hectáreas. Una ONG ambientalista alemana, Deutsche Umwelthilfe, ha advertido que "somos testigos de una pérdida de especies sin precedentes en el campo alemán. Especialmente aves como la tarabilla norteña, el hortelano, la perdiz gris o la golondrina de los bosques, pero también la golondrina común se ve afectada. El informe de DUH señala que las tierras marginales y de baja productividad, las que ahora se están volviendo a poner en producción, suelen tener una gran riqueza en biodiversidad. Según BirdLife International, "lo que estamos observando es una clara tendencia a la intensificación, lo que provocará grandes presiones sobre el medio ambiente. Una de las cuestiones más relevantes será la calidad del agua. Ya tenemos graves problemas de contaminación en los acuíferos y en los ríos de las regiones más cultivadas de Europa. ${ }^{9}$ El impacto en el suelo y en el carbono del suelo (es decir, en el clima) será previsiblemente igual de peligroso que el de la conversión de tierras del Programa de Reserva para la Conservación, especialmente en el caso de las tierras que han estado abandonadas varios años.

\section{Sueños de Jatrofa}

La jatrofa (Jatropha curcas) es un cultivo del que se está hablando como ideal para las tierras marginales de los países en desarrollo, es decir, los suelos pobres y con poca agua. Existen planes para implantar este cultivo en África, Asia y América del Sur. Sin embargo, no hay experiencias sobre su cultivo a gran escala y se sabe poco de ella, excepto que, aunque pueda sobrevivir a las sequías y en suelos pobres, es difícil que en tales condiciones, pueda producir una cosecha que merezca la pena. Se está prometiendo trabajo a millones de personas, pero la auténtica viabilidad de este cultivo es muy incierta.

Rajgopal, de la Universidad de California en Berkeley, indica que las producciones de jatrofa varían mucho dependiendo de la fertilidad del suelo y del agua. Plant Research

\footnotetext{
${ }^{7}$ Ducks Unlimited (2008), Carbon, Conservation Reserve Program and Native Prairie, abril de 2008.

${ }^{8}$ Joseph Fargione et al. (2008), "Land Clearing and the Biofuel Carbon Debt", Science, 29 de febrero de 2008. DOI: $10.1126 /$ science. 1152747 .

${ }^{9}$ BirdLife International. http://news.bbc.co.uk/1/hi/world/europe/7538102.stm
} 
International ha concluido en sus estudios que "si se tiene como objetivo una elevada producción de aceite, entonces no son ciertas las afirmaciones de que se requieren pocos nutrientes, poca agua, poca mano de obra, de que no supone una competencia para la producción de alimentos, de que resiste a las pestes y a las enfermedades". ${ }^{10}$

Combustibles de Agricultura en Tecnología Comunal (FACT), un centro de información basado en Holanda, llega incluso a advertir que se deben suspender las plantaciones a gran escala de jatrofa, por lo menos durante 4 o 5 años, hasta que se tengan suficientes datos experimentales que permitan evitar malas inversiones. "En este momento es importante distinguir entre 'realidad', 'promesas' y 'extrapolaciones peligrosas', ${ }^{11}$ advierten. La información que ofrecen indica que, para una producción óptima de aceite de jatrofa se requiere una gran pluviosidad de hasta 1000 o $1500 \mathrm{~mm} /$ hectárea, cantidades que están muy lejos de las precipitaciones de las tierras que se suelen considerar "marginales".

La evidencia sugiere que muchos inversores en agrocombustibles ya conocen estos datos y están optando por desarrollar sus plantaciones de jatrofa en tierras fértiles y bien irrigadas que para nada se pueden considerar "marginales".

\section{Agrocombustibles y apropiaciones de tierras}

En su informe de 2007 titulado "Los biocombustibles en África: su impacto sobre la tierra, los alimentos y los bosques", la organización African Biodiversity Network (ABN, Red Africana por la Biodiversidad) concluye que los casos de "apropiación de tierras" (en los que se privatizan grandes extensiones de tierra comunal) se han acelerado con la invasión de nuevas plantaciones para la producción de agrocombustibles. Un informe de una ONG local titulado "Biocombustibles y apropiaciones de tierras en el norte de Ghana" 12 denuncia también la facilidad con la que los inversores en agrocombustibles se apropian de las tierras comunales en las poblaciones rurales de África. Las comunidades afectadas rara vez son tenidas en cuenta en estos procesos, y desde luego no se las compensa por las expulsiones forzosas.

La especulación con las tierras está aumentando. La empresa Emergent Asset Managment, situada en el Reino Unido, acaba de lanzar su Fondo de Tierra Agrícola Africana, invitando a los inversores a participar en "el floreciente sector agrícola subsahariano". Las empresas con intereses comerciales están incluso utilizando los agrocombustibles como un medio de apoderarse de grandes extensiones de terreno. Una

\footnotetext{
${ }^{10}$ Plant Research International (2007), Reunión de expertos en Jatrofa, Bruselas, Dr. R.E.E. Jongschaap, diciembre de 2007.

${ }^{11}$ FACT (2007), Documento de posición sobre la Jatropha curcas, junio de 2007.

${ }^{12}$ RAINS/ABN (2008), Biofuel Land Grabbing in Northern Ghana, abril de 2008.
} 
vez que controlan la tierra, pueden decidir si la dedican a otros usos, como explotaciones madereras, mineras o incluso el turismo.

Podrían incluso esperar beneficiarse de los fondos destinados a los bosques que se cree que se establecerán en un futuro próximo bajo un acuerdo sobre el clima; o podrían beneficiarse también de Mecanismos de Desarrollo Limpio MDL. Sea como sea, una vez que se rompen las relaciones entre las poblaciones locales y sus tierras, es muy difícil recuperarlas. Cada año, cientos de miles de personas se ven obligadas a desplazarse para terminar viviendo en los suburbios de las ciudades, donde ya no podrán producir sus alimentos, sino que tendrán que buscar medios con los que comprarlos.

\section{¿Son los agrocombustibles beneficiosos para el desarrollo?}

Un argumento que se escucha con frecuencia a favor de la producción de agrocombustibles en tierras marginales es que contribuirá al desarrollo de los pobres en áreas rurales. La Red Africana por la Biodiversidad (ABN), en su carta de agosto de 2008 a los parlamentarios europeos ${ }^{13}$ a propósito del objetivo de la UE de llegar al $10 \%$ de agrocombustibles, decía:

"Negamos radicalmente el mito de que existen grandes extensiones de tierra improductiva en África. Los agricultores, los pastores y las comunidades indígenas usan las llamadas "tierras marginales", pero sus propios gobernantes no reconocen los derechos que tienen sobre ellas. El 70\% de las tierras de África son aún de propiedad comunal, lo que nos hace particularmente vulnerables a los proyectos de producción de biocombustibles que buscan privatizar la tierra y expulsar a las comunidades de unas tierras que son su medio de vida.

Negamos el mito de que la producción de biocombustibles a gran escala signifique desarrollo económico para los africanos. No podemos aceptar la promesa de crear unos pocos puestos de trabajo discontinuos y mal pagados para que se expulse a los agricultores de sus tierras, se deslocalice la producción de alimentos y se talen los bosques. A juzgar por los proyectos de biocombustibles que se han implementado en África hasta la fecha, éstos tendrán como consecuencia la expulsión a gran escala de los agricultores y pueblos indígenas, apropiaciones de grandes extensiones de terreno, subidas en los precios de los alimentos, deforestación, amenazas para las reservas de biodiversidad, pobreza y deudas para los pequeños agricultores; además, las tierras más ricas y mejor irrigadas dejarán de

\footnotetext{
${ }^{13}$ Red Africana por la Biodiversidad (2008), Renewable Energy Directive must not promote unsustainable biofuels, agosto de 2008.
} 
producir alimentos para producir cultivos destinados a combustibles.

...Ante la alarmante subida de los precios de los alimentos, que afecta principalmente a las zonas rurales empobrecidas de África, no estamos dispuestos a sacrificar los alimentos de nuestros pueblos para que los coches europeos sigan circulando."

Pocas comunidades están percibiendo que los cambios producidos por la producción a gran escala de agrocombustibles produzca verdaderos beneficios en su calidad de vida. La realidad es, con demasiada frecuencia, muy distinta a esa promesa dorada de empleos e ingresos para todos. Cuando las plantaciones de agrocombustibles tienen como consecuencia la conversión de sus pastos y tierras, la destrucción de los árboles que usan como fuente de alimentos y medicinas, y sólo unos pocos puestos de trabajo mal pagados en las tierras que antes eran suyas, los afectados descubren que no es a ellos a quienes beneficia el desarrollo.

\section{Conclusiones}

Se dice que la producción de agrocombustibles en tierras marginales traerá consigo el desarrollo para los países del Sur, pero se ocultan los efectos que ya han producido en los bosques, la seguridad alimentaria, el cambio climático y los derechos de propiedad sobre las tierras. Si estudiamos las implicaciones con más detalle concluiremos que este uso de las "tierras marginales" no evitará tales problemas, sino que los agravará.

La mayor parte de los debates en torno a las tierras "marginales" han ignorado la presencia en estas tierras de pastores, poblaciones indígenas, pequeños agricultores y mujeres; además, no se termina de entender que la agricultura intensiva y el monocultivo no son las únicas formas de explotar las tierras.

La promoción de proyectos de agrocombustibles en las tierras "marginales" provocará también un agravamiento de la pérdida de derechos de las comunidades indígenas. La pérdida de los modos de vida y de la seguridad alimentaria experimentada por los afectados desmiente el argumento de que los agrocombustibles puedan fomentar el desarrollo de las comunidades.

La conversión de pastos y tierras de reserva para la producción de agrocombustibles liberará mucho más carbono del que se pueden ahorrar con el uso de combustibles fósiles alternativos. Los cambios en la ecología de los paisajes acelerará los cambios climáticos a pequeña escala y hará aumentar la presión a la que se ven sometidas las comunidades locales.

Se desconoce el potencial real de las tierras de marginales secas para producir agrocombustibles a gran escala. 
Muchas empresas prefieren por eso establecerse en zonas de bosque, más fértiles y mejor irrigadas, aunque sigan afirmando que sólo usan tierras "marginales". En la mayor parte de los países afectados, los gobiernos carecen absolutamente de la disposición y la capacidad para asegurar que se protejan los derechos sobre las tierras, los ecosistemas amenazados y la seguridad alimentaria. La carrera de los agrocombustibles seguirá por lo tanto fomentando las apropiaciones de tierras de las comunidades locales.

Se han hecho peligrosas afirmaciones acerca de la extensión de las tierras "marginales" y su definición. Se necesitan nuevos estudios para determinar quién vive en esas tierras "marginales" y dónde, y qué extensiones de auténticas tierras de deshecho hay disponibles.

Muy pocas tierras son realmente "marginales". La tierra que es "marginal" para una persona puede ser un recurso vital para otra.

El mito de las tierras marginales no se puede utilizar para justificar dañinos proyectos de producción de agrocombustibles.

\section{Anexo 4 estudios de caso}

\section{Estudio de caso 1: ¿Qué significa «tierra marginal” en Indonesia?}

Según el gobierno de Indonesia hay tres tipos de tierra que no se usan para la agricultura: tierra marginal, tierra crítica y tierra dormida. En Indonesia, la tierra "marginal" es tierra no productiva con un alto nivel de acidez. Por lo tanto la tierra marginal de Indonesia incluye terrenos pantanosos, humedales y bosques de turba, así como tierras ácidas secas, como las áreas montañosas o las islas del este, que presentan condiciones similares a las de Australia.

La tierra "crítica" es la que se ha degradado desde el punto de vista ecológico como resultado de la agricultura intensiva y que ya no es válida para su cultivo. Estas tierras degradadas fueron en el pasado importantes para mantener la seguridad alimentaria en el país, y se debería dar prioridad al mantenimiento de esta seguridad mediante, por ejemplo, la mejora de los sistemas de riego.

Tierra "dormida" es la que permanece temporalmente sin cultivar, pero es extraordinariamente importante para las comunidades indígenas que combinan cultivo y barbecho con la caza.

Según Marianne Klute, de Watch Indonesia, "Si los políticos hablan de tierras marginales, el gobierno indonesio puede tomarlo como una luz verde para talar más bosques de turba. Recomendar la producción de agrocombustibles en las tierras críticas sería una irresponsabilidad, especialmente cuando estas tierras se necesitan para la 
producción de alimentos. Y recomendar que se produzcan agrocombustibles en las tierras dormidas sería una incitación irresponsable a la expulsión de granjeros, cazadores y poblaciones indígenas".

\section{Estudio de caso 2: Kalimantan Occidental, Indonesia}

Icin, un indígena Dayak de Kalimantan Occidental, al contemplar un paisaje variopinto de campos deforestados, tierras degradadas, bosques secundarios y plantaciones de palma para la producción de aceite, comenta: "Estas eran nuestras tierras. Y ya las hemos perdido dos veces. Primero, hace unos veinte años, las compañías madereras comenzaron a talar los bosques".

Solo un $25 \%$ de las tierras que entonces se desmontaron se convirtieron en plantaciones de palma, que son propiedad del estado. Las comunidades del distrito de Sanggau sobrevivieron en los bosques que quedaron, pero también en las tierras deforestadas que dedicaron al barbecho y los cultivos rotativos; plantan arroz y maíz los dos primeros años, alternándolos con caucho y frutales. Tras el segundo año, se dejan descansar las plantaciones nuevas de caucho. En la actualidad, para satisfacer la demanda mundial de energía, la compañía estatal de aceite de palma PTPNXIII planea ampliar sus plantaciones a la gran parte de su concesión que aún no está sembrada con palma, que se considera tierra improductiva y dormida.

"Hace ocho años perdimos incluso más de nuestras tierras a favor de una compañía privada de aceite de palma. Una noche las excavadoras destruyeron las plantaciones de caucho e incluso nuestros arrozales. Desde entonces, se han talado la mayor parte de los bosques primarios que quedaban", nos dice Icin mientras contempla la tierra desnuda que se extiende hasta las montañas.

Desde el año 2000, la tala se ha incrementado a una tasa nunca vista. La madera se vende a Malasia y, en la actualidad, dos tercios de Kalimantan Occidental está en manos de las compañías de aceite de palma. El Grupo Ganda, que tiene estrechas relaciones con el grupo Wilmar ha establecido una plantación en las tierras que Icin utilizaba para producir caucho. Pero la mayor parte de lo que una vez fueron bosques comunales tradicionales ya destruidos queda como tierras sin explotar, degradados y erosionados. Condenadas a la pobreza sin sus bosques, las comunidades tratan de cultivar el suelo erosionado.

“QQue nos pasará a nosotros?”, se pregunta Icin. "El gobierno tiene ambiciosos planes para establecer nuevas plantaciones a gran escala". Señala en un mapa la futura plantación de Sinar Mas, en tierras supuestamente improductivas y "dormidas". "En realidad, siete aldeas deberían estar señaladas en esta zona, pero ni están mencionadas. ¿Significa esto que ya no existimos para el resto del mundo?” 


\section{Estudio de Caso 3: Bosques del Chaco, Argentina}

Mientras los precios de la soja siguen aumentando en todo el mundo, la producción de esta oleaginosa se ha extendido más allá de las zonas agrícolas tradicionales para ocupar "tierras marginales" en el norte del país. Las víctimas han sido los campesinos que cultivan cereales destinados a la alimentación humana y las comunidades indígenas que luchan por sus derechos sobre las tierras ancestrales de las que dependen para subsistir. Se trata de una de las zonas más ricas en biodiversidad del país.

Cerca de Embarcación, en la provincia de Salta, una comunidad indígena Wichi resiste contra la deforestación provocada por los monocultivos de soja de los bosques secos del Chaco. Además, los productores de soja ya consiguieron expulsar violentamente de sus tierras a la comunidad indígena guaraní Jase Endy Guasu, en el Talar, en la vecina provincia de Jujuy.

Se prevé que los violentos conflictos de tierras motivados por la soja continúen debido a los planes de algunos departamentos del gobierno de introducir además plantaciones de jatropha para extender los cultivos destinados a la producción de agrocombustibles aumentando así los conflictos por la tierra.

\section{Estudio de Caso 4: Wolaita, Ethiopia}

Wolaita, en el Sur de Etiopía, está ahora en el punto de mira de los nuevos desarrollos de agrocombustibles. Según la ONG local Eco Yeshemachoch Mahiber, la compañía británica Sun Biofuels se ha apropiado de 3000 hectáreas de pastos comunales para establecer una plantación de Jatrofa. Global Energy Ethiopia está cultivando ricino en 2350 hectáreas mediante contratos con pequeños agricultores.

Tanto el gobierno etíope como las empresas han declarado que la producción de cultivos para agrocombustibles sólo se llevará a cabo en tierras marginales, abandonadas o improductivas, lo que contrasta de lleno con los testimonios de la población local. Según los agricultores de la zona, las plantaciones se están estableciendo en los pastos comunales y en las tierras de cultivo más fértiles.

Se trata de una de las zonas más densamente pobladas del país; entre 360 y 700 personas por kilómetro cuadrado. Como resultado de esta intensa competición por la tierra, la zona no puede asegurar alimentos para su población. Alrededor de un 36\% de ésta en las áreas en las que se han planificado estas explotaciones depende de las ayudas alimentarias de emergencia o del programa "Alimentos por Trabajo".

Es en este escenario que la producción de agrocombustibles ha reemplazado la muy necesaria producción de alimentos y pastos. Combinado con la devastadora crisis 
alimentaria, esto ha motivado que los precios de los alimentos se disparasen en esta zona, hasta quintuplicar los precios del año pasado.

Las compañías insisten en que los beneficios de la producción de biocombustibles contribuirán a mejorar el nivel de vida de las comunidades, pero los granjeros denuncian que las empresas aún no han pagado lo prometido y ni siquiera les han permitido ver una copia de sus contratos.

Con la caída en la producción de alimentos y la subida de los precios, la comunidad se encuentra más insegura alimentariamente que nunca, agravada esta situación por la producción de agrocombustibles en estas tierras supuestamente "improductivas". 


\section{Bibliografía}

Berndes, Goeran et al. (2003), «The contribution of biomass in the future global energy supply: a review of 17 studies», Biomass and Bioenergy, 25 (2003), 1-28, 1 de julio de 2003.

CLIP (ILRI, MSU) (2008), The Effects of Climate and Land Use Changes on Climate and Agricultural Systems in Kenya, junio de 2008.

Ducks Unlimited (2008), Carbon, Conservation Reserve Program and Native Prairie, abril de 2008.

Environmental Defense Fund factsheet (Circular del Fondo de Defensa Medioambiental), 2008.

http://www.nativeecosystems.org/documents/CRPFactSheet

Fargione, Joseph et al. (2008), «Land clearing and the biofuel carbon debt» en Science, 29 de febrero de 2008. DOI: 10.1126/science.1152747

FACT (2007), Documento de posición sobre la Jatropha curcas, junio de 2007.

FAPRI (2007), "Estimating Water Quality, Air Quality and Soil Carbon Benefits of the Conservation Reserve Program", Facultad de Agricultura, Alimentación y $\begin{array}{llll}\text { Recursos Naturales, enero } & \text { de } & \end{array}$ http://www.fsa.usda.gov/Internet/FSA_File/606586_hr.pdf

Field, Christopher B. et al. (2008), «Biomass energy: the scale of the potential resource» en Trends in Ecology and Evolution, volumen 23, $\mathrm{n}^{\circ}$ 2, febrero de 2008.

The Gallagher Review of the indirect effects of biofuel production (2008), Agencia de Combustibles Renovables, Julio 2008.

RAINS/ABN (2008), Biofuel Land Grabbing in Northern Ghana, abril de 2008.

Rajgopal, Deepak (2007), Rethinking Current Strategies for Biofuel Production in India, Universidad de California, Berkeley.

Red Africana por la Biodiversidad (2007), Agrofuels in Africa: the impact on land, food and forests, julio de 2007. 
Red Africana por la Biodiversidad (2008), Renewable Energy Directive must not promote unsustainable biofuels, agosto de 2008.

Rossi, Andrea y Lambrou, Yianna (2008), Gender and Equity Issues in Liquid Biofuels Production - Minimising the Risks to Maximise the Opportunities, FAO, abril de 2008.

Royal Society (2008), Sustainable biofuels: prospects and challenges, Royal Society, 14 de enero de 2008.

Tilman, David et al. (2006), «Carbon-Negative Biofuels from Low-Input HighDiversity Grassland Biomass» en Science, 8 de diciembre de 2006, DOI: 10.1126/science. 1133306 . 